Review Article

\title{
The Value of Information
}

\author{
Martin J Vilela* \\ Aura Energy Solutions, Spain
}

\begin{abstract}
Value of information is a methodology that aims to determine whether it is worth acquiring new information. The acquisition of new data may reduce the uncertainties of the input parameters that define the value of a project. Such a reduction can increase our expectations and consequently the value of the project. The acquisition of data is justifiable only if their cost is less than the project value increases due to an increase in the certainty of the parameters. This article reviews the concepts and methodology of the value of information when the uncertainty is due to a lack of information and discusses an example of this methodology. A decision tree is built for the ideal perfect and imperfect information cases in the example shown and both results are contrasted. It is shown that, in the example discussed, it is worth acquiring the data if their cost is less than the calculated threshold. This article also introduces an additional form of uncertainty that occurs because of ambiguity and will be discussed in a future article.
\end{abstract}

Keywords: Methodology, Acquiring the data, VOI

\section{The Rationale}

One of the most important tasks we do every day is make decisions. These decisions may be as trifling as the shoes to wear today or as important as investing all our resources (or our company resources) in a risky venture. In very few cases, decisions are made in a certain environment (e.g. we may know that right now it is raining, so there is no doubt we need to carry an umbrella). However, in the majority of the cases, decisions are made with uncertainties. Uncertainties are the consequence of a lack of information on current or future states of nature. During subsurface assessments, geoscientists integrate field-scale information (such as seismic data that gives interpreted information (subjective) of the reservoir structure) with well-scale information (such as logs and core data that provides information and interpreted information on the well porosity, fluid properties, saturation, permeability, etc.) All this data, either seismic or well data, carry uncertainties. These uncer- tainties are much more significant in the inter-well space, where the data is estimated using interpolation methods.

To decide whether a project should be sanctioned, we must predict future production scenarios, costs, hydrocarbon prices, and other parameters. These predictions are used to assess the benefits of the project versus the associated cost. The predictions of future reservoir performance are made with deterministic models, which operate on a set of input parameters and generate outcomes. Different sets of inputs generate various realisations of the same reservoir and, consequently, different outcomes, which can be statistically treated. There are cases where additional information can be acquired to reduce the uncertainty in the reservoir parameters. However, acquiring data has a cost, either for acquiring the data itself or due to delay in the project execution while data is obtained.

The intention of acquiring data is to totally or partially resolve the uncertainty of the parameters. For example, during a reser-

\begin{tabular}{|l|l|}
\hline \hline Quick Response Code: & *Corresponding author: Martin J Vilela, Aura Energy Solutions, Spain \\
Received: 26 August, 2021 & \multicolumn{1}{c|}{ Published: 14 September, 2021} \\
& Citation: Vilela MJ. The Value of Information. Trends Petro Eng. 2021;1(2):1-5. DOI: \\
10.53902/TPE.2021.01.000507
\end{tabular}


voir's exploration phase, we can assume a particular oil composition based on an analogue field; however, this assumption carries uncertainties. To resolve the uncertainty, we can take a bottom hole sample and get the oil composition in a selected place of the reservoir. Based on the geological understanding of the reservoir, this oil composition can either be assumed for the complete reservoir, or we may need additional samples in other areas of the reservoir. The current valuation of projects that will be executed in the future is obtained using expectations. Expectations are calculated using the estimated chances associated with the possible realisations of the reservoir. The value of information (VOI) is the methodology for assessing the value that acquiring new data adds to the project value.

\section{The History}

The oil and gas industry likely is one of the most funds-demanding industries. Many multi-million projects are sanctioned every year, and this situation has been so since the beginning of the last century. For those projects, options to acquire additional data are often possible. Schlaifer, ${ }^{1}$ Raiffa and Schlaifer ${ }^{2}$ and Raiffa ${ }^{3}$ developed the fundamental concepts and tools of VOI in the context of business administration; their goal was to enable business administrators to make wiser decisions. Their approach consists of using statistical inference and sampling tools in practical decision-making problems under uncertain conditions, where additional information about the state of the world can be obtained through experimentation. At the same time, Grayson ${ }^{4}$ published his dissertation (converted into a book the same year), applying the VOI methodology to drilling decisions made by oil and gas operators, where uncertainties are exceptionally great. Grayson's work is the first reference showing the use of utility theory and subjective probability theory applied to an oil and gas decision problem that the author of this paper is aware of. Grayson ${ }^{5}$ used statistical inference nomenclature to analyse the drilling decision and the value associated with gathering additional information.

The next milestone for VOI in the oil and gas industry is the $\mathrm{PhD}$ thesis of Newendorp, ${ }^{6}$ who discussed the necessity of developing and using the risk attitude of the decision-maker as part of the VOI assessment; he specifically considers the use of the exponential utility function to capture the decision maker's risk attitude. Newendorp ${ }^{7}$ discussed the logic, mathematical proof, and methodology of Bayes' theorem (developed by Thomas Bayes in 1763) in detail, which is a fundamental mathematical tool behind VOI. He also discussed the concept of sequential sampling or sequential data acquisition. Subsequently, further research and applications expanded the scope of the subject and provided more robustness to the methodology. Since the 1970s, several developments and applications have been published that strengthen the VOI methodology (see reference for examples). Despite all of the applications reported for this methodology, VOI is still far from being a common practice in most exploration and production companies. ${ }^{8}$

\section{The Concepts}

\section{Perfect vs imperfect data}

Data is called perfect when it can accurately and precisely predict the value of the uncertain variables it is expected to resolve. This kind of data, called clairvoyant, is just a concept because data can never predict the actual value of variables without some degree of uncertainty. However, perfect data is essential because it helps estimate the maximum increase in project value that it can add. However, real data is imperfect and, as such, may only partially resolve the uncertainty in the associated variable. ${ }^{9}$ For example, the Original Oil in Place in a reservoir is a function of several parameters, and reservoir thickness is one of them. If a few wells have already been drilled, then they are used to estimated reservoir thickness. However, that estimate has uncertainty because the thickness can change in other areas of the reservoir. Additional wells can be drilled to reduce that uncertainty in areas where there is no data or data is scarce.

\section{Probability vs fuzzy logic}

Probability concepts are associated with Boolean logic: an element either belongs to a set or not. The degree of belonging is totally or none. Probability is the tool we use to handle uncertainty related to a lack of information. Because we are uncertain about the value of the variables, we describe their uncertainty using a discrete number of states, usually three discrete states: high, medium and low; the probabilities of the three states should add up to 1.0. These probabilities represent the chances that the actual state corresponds to each of the discrete states.

When a Monte Carlo simulation is used to represent the uncertainty of the variables, the cumulative probability should add up to 1.0. Fuzzy logic is the tool used to describe ambiguity, a different type of uncertainty than the lack of information. Ambiguity happens when an element only partially belongs to a set. In this case, one element can belong partially to more than one set. Ambiguity is described using fuzzy logic and its magnitude through the membership functions. ${ }^{10}$

\section{Money vs utility}

Most of the time, projects are evaluated using money. Of course, the logic of that valuation is that more money is better. However, this description considers neither the risk attitude of the decision-maker nor the asymmetric preferences. Typically, decision-makers are not neutral to money losses or gains. The risk attitude is captured using a utility function that translates money to what matters to the decision-maker (utility) and incorporates the asymmetry in the valuations. ${ }^{11}$

\section{The Methodology}

The value of information is the methodology that compares the 
value of a project as it is (without consideration given to data acquisition) with the value of the same project when data is acquired (which should increase our knowledge of the true value of the pa-

\section{VOI $=$ project's value with new information - project's value without new information}

If the outcome of this equation is positive, there is a benefit (increase in the project's value) from acquiring the data. Data acquisition is recommended when the cost associated with the data is lower than the VOI; otherwise, data acquisition reduces project value.

These concepts will be illustrated with a simple example. For the example considered, Table 1 shows the project value and the associated prior probabilities of three selected states of nature: high, medium, and low. These states of nature capture the uncertainty in the project's value; it is also included the reliability and posterior probabilities. The domain experts' team members estimate the prior and reliability probabilities based on their experience and knowledge; posterior probabilities are calculated, reversing the reliability probabilities using Bayes' theorem.

In Table 1, for describing the conditioned probabilities, capital letters represent states of nature $(\mathrm{H}=$ high, $\mathrm{M}=$ medium, $\mathrm{L}=\mathrm{low})$, and small letters represent the outcome of the data acquisition ( $h=h i g h$, $\mathrm{m}=$ medium, l=low).

Reversing the reliability probabilities is required because the conditional probabilities that we can estimate, based on our knowledge, are the probabilities of a data outcome knowing the states of nature. However, the decision's logic requires estimating the probability of the states of nature knowing the data outcome. ${ }^{12}$

Figure 1 shows the decision tree representing the VOI of our rameters related to the data acquisition). VOI is described by the equation below:

project, assuming that the information is perfect to the left-hand side and the information is imperfect to the right-hand side. In both cases, at the top of the trees, we have the no data acquisition case and, at the bottom, the data acquisition case.

Let us assume that information is perfect (left-hand side of Figure 1). In this case, when data is acquired to support the prediction of the states of nature, the value of the project increases from $\$ 100 \mathrm{M}$ to $\$ 193 \mathrm{M}$. This means that the project resulting from the acquired data produces more benefits than the project without data. ${ }^{13-15}$ Under the assumption that information is perfect, the maximum cost we can afford to acquire data without reducing project value is $\$ 93 \mathrm{M}$. This result encourages the analysis of the imperfect information case. The imperfect information case is shown on the right-hand side of Figure $1 .{ }^{15-20}$ The uncertainty in the data, captured by the reliability probabilities shown in Table 1 , reduces the value of the data from $\$ 193 \mathrm{M}$ to $\$ 109 \mathrm{M}$.

This result means that when imperfect information is used to support the prediction of the state of nature, data acquisition adds value to the project as long as the cost of it is less than $\$ 9 \mathrm{M}$, which is the difference between the project with imperfect information and the project without data. Otherwise, if data costs are higher than $\$ 9 \mathrm{M}$, data acquisition is not recommended, and the best option is to decide on the project's best way forward based on current information..$^{20-26}$

Table 1: State of nature, prior, reliability and posterior probabilities.

\begin{tabular}{|c|c|c|c|c|c|c|c|c|}
\hline \multicolumn{3}{|c|}{ Project value / State of nature } & & & & & & \\
\hline High, M\$ & Medium, M\$ & Low, M\$ & & & & & & \\
\hline 700 & 100 & -400 & & & & & & \\
\hline \multicolumn{3}{|c|}{ Prior probabilities } & & & & & & \\
\hline High & Medium & Low & & & & & & \\
\hline 0.25 & 0.45 & 0.30 & & & & & & \\
\hline \multicolumn{9}{|c|}{ Reliability probabilities } \\
\hline $\operatorname{Prob}(h \mid H)$ & $\operatorname{Prob}(\mathrm{m} \mid \mathrm{H})$ & $\operatorname{Prob}(1 \mid \mathrm{H})$ & $\operatorname{Prob}(\mathrm{h} \mid \mathrm{M})$ & $\operatorname{Prob}(\mathrm{m} \mid \mathrm{M})$ & $\operatorname{Prob}(1 \mid \mathrm{M})$ & $\operatorname{Prob}(\mathrm{h} \mid \mathrm{L})$ & $\operatorname{Prob}(\mathrm{m} \mid \mathrm{L})$ & $\operatorname{Prob}(1 \mid \mathrm{L})$ \\
\hline 0.50 & 0.30 & 0.20 & 0.20 & 0.60 & 0.20 & 0.10 & 0.20 & 0.70 \\
\hline \multicolumn{9}{|c|}{ Posterior probabilities } \\
\hline $\operatorname{Prob}(\mathrm{H} \mid \mathrm{h})$ & $\operatorname{Prob}(M \mid h)$ & $\operatorname{Prob}(L \mid h)$ & $\operatorname{Prob}(H \mid m)$ & $\operatorname{Prob}(M \mid m)$ & $\operatorname{Prob}(\mathrm{L} \mid \mathrm{m})$ & $\operatorname{Prob}(\mathrm{H} \mid \mathrm{l})$ & $\operatorname{Prob}(M \mid 1)$ & $\operatorname{Prob}(\mathrm{L} \mid \mathrm{l})$ \\
\hline 0.51 & 0.37 & 0.12 & 0.19 & 0.67 & 0.15 & 0.14 & 0.26 & 0.60 \\
\hline
\end{tabular}



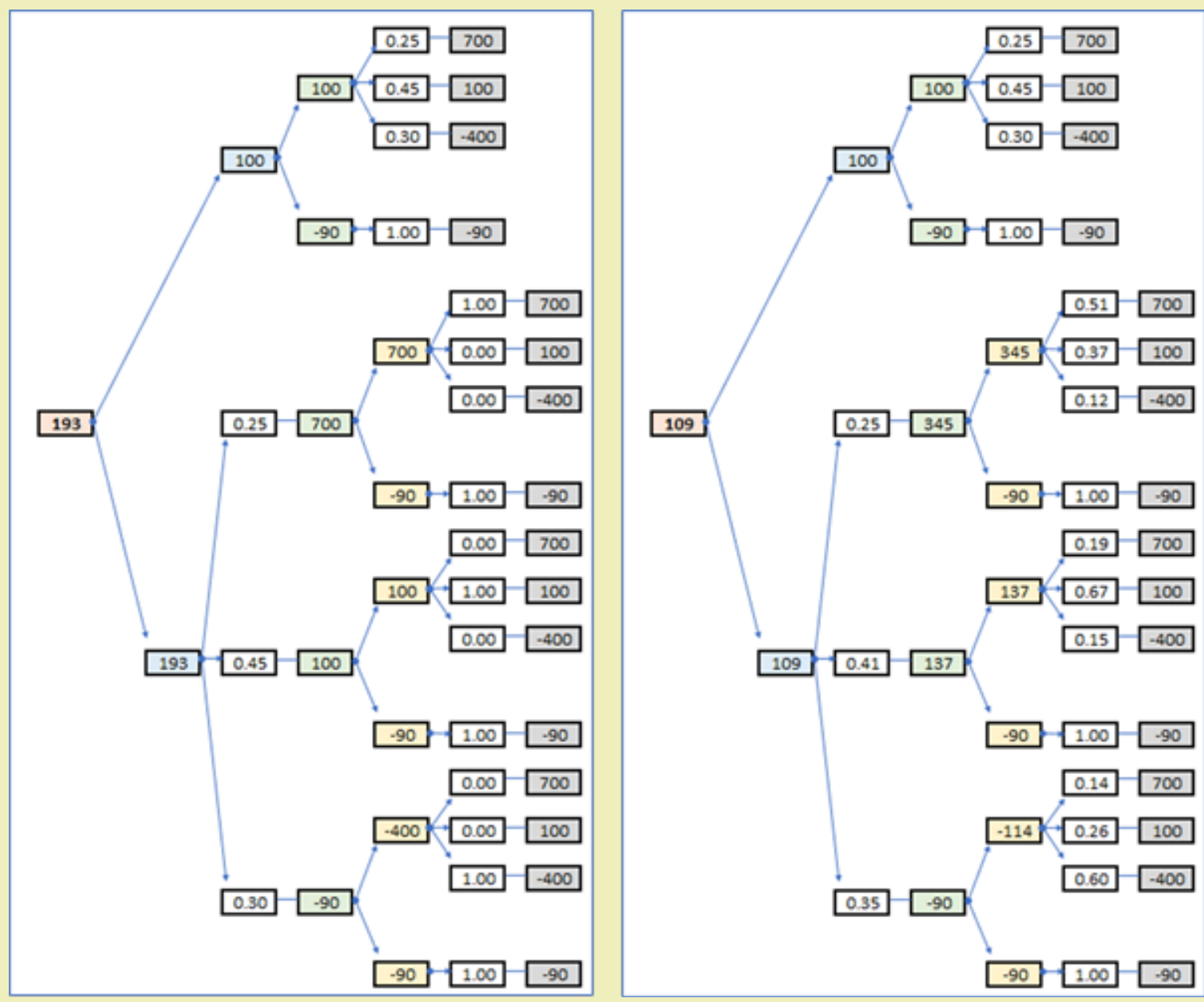

Figure 1: Decision trees for perfect and imperfect data.

\section{The Conclusion}

Data acquisition may increase project value by partially resolving some of the uncertainties and, consequently, changing our expectations of the uncertain project outcome, impacting its value. However, the cost of the data should always be less than VOI to avoid spoiling the project value. Data can only have value if it can change a decision that otherwise would be different; there is no value in uncertainty reduction but project value creation.

Even though perfect information does not exist, the value of perfect information provides the maximum benefit that data can add to the project value. If the value of perfect data discards the option of data acquisition, the VOI assessment is concluded without estimating the imperfect information case. In the example shown, we first demonstrated that the value of perfect data acquisition, which is the same as the value associated with removing the uncertainties, is $\$ 93 \mathrm{M}$. However, the value of the imperfect data acquisition, a realistic case, is $\$ 9 \mathrm{M}$. This means that data is recommended to be acquired as long as its cost is less than $\$ 9 \mathrm{M}$. In this article, we discuss the methodology of the VOI, considering the uncertainty due to a lack of information, which we call the standard VOI methodology.
This assessment requires the use of probability theory. The uncertainty due to fuzziness in the data is not discussed here, but it will be the subject of another article.

\section{Acknowledgments}

None.

\section{Funding}

This work had no funding of any kind.

\section{Conflicts of Interest}

Author declares that there is no conflict of interest.

\section{References}

1. Schlaifer R. Analysis of decisions under uncertainty. New York, NY, USA: McGraw-Hill; 1959.

2. Raiffa H, Schlaifer R. Applied statistical decision theory. Boston, Massachusetts, USA: Harvard University, 1961.

3. Raiffa H. Decision analysis: Introductory Lectures on-Choices Under Uncertainty. Reading, Massachusetts, USA: Addison-Wesley; 1968.

4. Grayson CJ. Decisions Under Uncertainty. Drilling Decisions by Oil and Gas Operators. Boston, Massachusetts, USA: Harvard Business School University, Division of Research, 1960. 
5. Grayson CJ. Bayesian Analysis. A New Approach to Statistical Decision-Making. Paper 266 presented at the SPE Economics and Evaluation Symposium, Dallas, Texas, USA, 1962.

6. Newendorp P. Application of utility theory to drilling investment decisions. Doctoral thesis. Oklahoma, USA: The University of Oklahoma. 1967.

7. Newendorp P. Bayesian analysis-A method for updating risk estimates. Paper 3463 presented at the SPE $46^{\text {th }}$ Annual Fall Meeting, New Orleans, USA, 1971.

8. Bickel J, Bratvold R. Decision Making in the Oil and Gas Industry: From Blissful Ignorance to Uncertainty-Induced Confusion. Paper SPE 109610 presented at the Annual Technical Conference and Exhibition, Anaheim, California, USA, 2007.

9. Clemen R. Making Hard Decisions. An Introduction to Decision Analysis. $2^{\text {nd }}$ Edition. California, USA: Duxbury Press; 1996.

10. Vilela M, Oluyemi G, Petrovski A. Fuzzy Logic applied to Value of Information Assessment in Oil and Gas Projects. Petroleum Science. 2019;16(1):1208-1220.

11. Newendorp P, Schuyler J. Decision Analysis for Petroleum Exploration, $2^{\text {nd }}$ edition. Aurora, Colorado, USA: Planning Press; 2002.

12. Bratvold R, Bickel J, Lohne H. Value of Information in the Oil and Gas Industry: Past, Present, and Future. Paper 110378 presented at the Annual Technical Conference and Exhibition, Anaheim, California, USA, 2007.

13. Begg S, Bratvold R. The Value of Flexibility in Managing Uncertainty in Oil and Gas Investments. Paper SPE 77586 presented at the Annual Technical Conference and Exhibition, San Antonio, Texas, USA, 2002.

14. Coopersmith E, Cunningham P. A Practical Approach to Evaluating the Value of Information and Real Option Decisions in the Upstream Petroleum Industry. Paper 77582 presented at the Annual Technical Conference and Exhibition, San Antonio, Texas, USA, 2002.

15. Demirmen F. Use of Value of Information Concept in Justification and Ranking of Subsurface Appraisal. Paper 36631 presented at the Annual Technical Conference and Exhibition, Denver, Colorado, USA, 1996.
16. Dougherty E. The oilman's primer on statistical decision theory. Paper 3278 of the Society of Petroleum Engineers Library (unpublished). 1971.

17. Dunn M. A Method to Estimate the Value of Well Log Information. Paper 24672 presented at the Annual Technical Conference and Exhibition, Washington, DC, USA,1992.

18. Gerhardt J, Haldorsen H. On the value of information. Paper 19291 presented at the Offshore Europe 89, Aberdeen, UK, 1989.

19. Koninx J. Value of information. From cost cutting to value creation. Paper 64390 presented at the Asia Pacific Oil Conference and Exhibition, Brisbane, Australia, 2002.

20. Lohrenz J. Net values of Our Information. Journal of Petroleum Technology. 1988;40(4):499-503.

21. Moras R, Lesso W. MacDonald R. Assessing the Value of Information Provided by Observation Wells in Gas Storage Reservoirs. Paper 17262-MS of the Society of Petroleum Engineers Library (unpublished). 1987.

22. Rose P. Dealing with risk and uncertainty in exploration: how can we improve? The American Association of Petroleum Geologists Bulletin. 1987;71(1):1-16.

23. Silbergh M, Brons F. Profitability Analysis-Where are We Now? Paper 2994 presented at the SPE $45^{\text {th }}$ Annual Fall Meeting, Houston, Texas, USA, 1970

24. Stibolt R, Lehman J. The Value of a Seismic Option. Paper 25821 presented at the Hydrocarbons Economics and Evaluation Symposium, Dallas, Texas, USA, 1993.

25. Suslick S, Schiozer D. Risk analysis applied to petroleum exploration and production: an overview. Journal of Petroleum Science and Engineering. 2004;44(2):1-9.

26. Warren J. Development Decision: Value of Information. Paper 11312 presented at the 1983 Hydrocarbon Economics and Evaluation Symposium of the Society of Petroleum Engineers of AIME, Dallas, Texas, USA, 1983 